\title{
Effects of compositional and rheological stratifications on small-scale convection under the oceans: Implications for the thickness of oceanic lithosphere and seafloor flattening
}

\author{
J. C. Afonso, ${ }^{1}$ S. Zlotnik, ${ }^{1}$ and M. Fernàndez ${ }^{1}$
}

[1] Pressure-release melting at mid-ocean ridges generate compositional and rheological layering in the oceanic mantle that may control the evolution of the oceanic lithosphere. We use dynamic models coupled with melting and petrological models to explore 1) the influence of this layering on the development of small-scale convection under the oceans, 2) its role in determining the thickness of oceanic lithosphere, and 3) its feasibility as responsible for the deviations of seafloor and surface heat flow from predictions by conductive models in mature oceanic lithosphere. Here we show that the existence of smallscale convection is entirely compatible with experimental creep parameters and flow laws, and that the viscosity stratification due to melt extraction (i.e., $\mathrm{H}_{2} \mathrm{O}$ removal) is the main factor controlling the plate's thermal evolution, its asymptotic thickness, and the flattening of seafloor and surface heat flow at ages $\gtrsim 70 \mathrm{Ma}$. The effects of Al-rich phase transitions and compositional layering are minor. Citation: Afonso, J. C., S. Zlotnik, and M. Fernàndez (2008), Effects of compositional and rheological stratifications on smallscale convection under the oceans: Implications for the thickness of oceanic lithosphere and seafloor flattening, Geophys. Res. Lett., 35, L20308,

\section{Introduction}

[2] It is widely accepted that the seafloor topography and surface heat flow in oceanic lithosphere (OL) younger than 70-80 Ma can be explained as the cooling of a semi-infinite half-space medium (i.e., half-space cooling model). On the contrary, the physical mechanism responsible for the deviations of these two observables from predictions by the halfspace cooling model in older OL (commonly refer to as flattening) has been the subject of one of the longeststanding debates in geophysics. Suggested mechanisms include: age-dependent crustal production, dynamic pressure related to asthenospheric flow, phase changes, chemical buoyancy of the mantle residuum, internal radiogenic heating, thermal rejuvenation by hot-spots, and small-scale convection (SSC) [cf. Hillier and Watts, 2005; Huang and Zhong, 2005, and references therein]. Although in principle all of these mechanisms could play a role, SSC has become one of the most popular mechanisms for explaining the deviations due to its sound physical explanation. Despite this general acceptance, most of the studies on SSC have

\footnotetext{
${ }^{1}$ Group Dynamics of the Lithosphere, Institute of Earth Sciences "J. Almera," CSIC, Barcelona, Spain.
}

been focused on the general conditions for the existence of it and on its associated onset times [e.g., Davaille and Jaupart, 1994; Solomatov and Moresi, 2000; Huang et al., 2003; Dumoulin et al., 2005, and references therein]. Specific numerical models devised to asses its feasibility as responsible for the flattening of seafloor and surface heat flow are only a few [Dumoulin et al., 2001; Korenaga and Jordan, 2002; Huang and Zhong, 2005; Zlotnik et al., 2008]. These studies consider the OL to be the cold thermal boundary layer above a convective adiabatic mantle, and therefore all physical properties are mainly controlled by the thermal structure. However, the OL is not only a thermal, but also a chemical and a mechanical boundary layer. Its thermodynamic properties, which in turn control its dynamic behaviour, depend ultimately on temperature, pressure, composition of the original source (i.e., upper mantle that has not experienced partial melting), and degree of melt depletion experienced at the MOR [e.g., Hirth and Kohlstedt, 1996; Phipps Morgan, 1997; Lee et al., 2005; Afonso et al., 2007]. While all these factors are intimately related, their influence on the generation of SSC and the evolution of OL have never been systematically explored with thermomechanical models. A relevant exception is the recent $3 \mathrm{D}$ numerical study of Ballmer et al. [2007], who included compositional effects on the density calculation to study intraplate volcanism in OL. Yet, rheological stratifications due to melt extraction and the expression of SSC on geophysical observables were not explicitly considered.

[3] In this paper, we present the first consistent assessment of the effects of rheological and compositional layering on the development and vigour of SSC under the oceans and its ability to generate the flattening of seafloor and surface heat flow in mature OL. We employ dynamic models coupled with MOR melting models, experimental parameterizations of rheologies affected by dehydration beneath MORs, and petrological models of OL.

\section{Numerical Model and Methods}

[4] Numerical solutions of the temperature and flow fields are obtained solving the two-dimensional equations of mass, momentum, and energy conservation in an infinite Prandtl number fluid with variable viscosity under the extended Boussinesq approximation (see details in the auxiliary material ${ }^{1}$ ). The thickness of the numerical domain represents $660 \mathrm{~km}$ in nature. The horizontal dimension is either 7000 or $15000 \mathrm{~km}$ wide, depending on the particular 


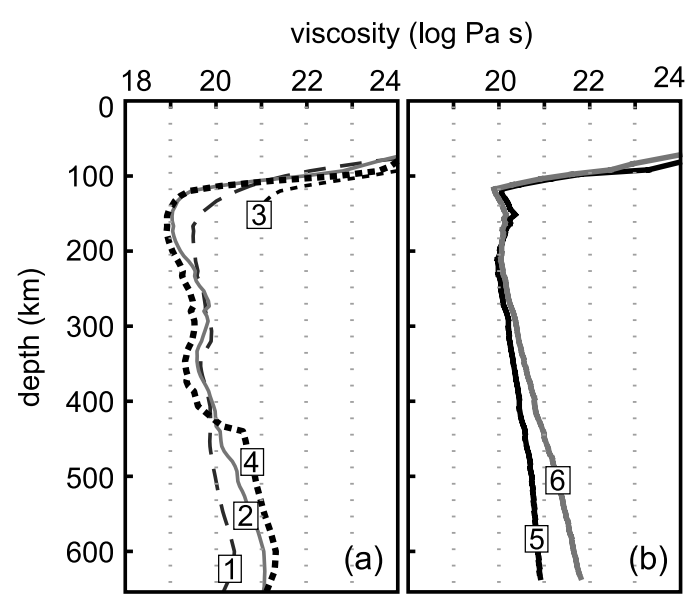

Figure 1. Typical viscosity profiles from our "wet" simulations. (a) Labeled curves denote: 1, viscosity associated with diffusion creep; 2, viscosity associated with dislocation creep; 3 , "dry" component of effective viscosity (i.e., $\eta_{d r y}=100 \eta_{\text {wet }}$ ); 4 , effective viscosity of the mantle calculated with 1, 2, and 3 as explained in the text. Note the dominant role of dislocation creep in the first $\sim 200 \mathrm{~km}$. A 10 -fold viscosity increase is imposed at $410 \mathrm{~km}$. (b) Typical effective viscosity profiles from our "dry" simulations using two different sets of "dry" parameters: 5, [Karato and Wu, 1993]; 6, [Hirth and Kohlstedt, 2003]. Note that the resulting viscosity is $\gtrsim 10^{20} \mathrm{~Pa} \mathrm{~s}$ in the entire upper mantle.

model. The effective viscosity of the fluid is computed as the harmonic mean of the two creep mechanisms that act simultaneously in the mantle, namely diffusion and dislocation creep (Figure 1a). The heat capacity $(C p)$ is taken constant, while the thermal conductivity $(k)$ and coefficient of thermal expansion $(\alpha)$ are $T$ - $P$-dependent. Internal heating is explicitly included in the energy equation and assumed to be composed of three terms: i) a constant radiogenic heat production term, ii) an adiabatic heating term, and iii) a shear heating term associated with mechanical heat dissipation.

[5] Temperature boundary conditions are such that the temperature is fixed at the top $\left(T_{\text {surf }}=273 \mathrm{~K}\right)$ and bottom $\left(T_{\text {bot }}=1880 \mathrm{~K}\right)$ of the numerical domain, while on the laterals the normal heat flow is set to zero. Unless indicated otherwise, the initial temperature field follows the halfspace cooling model. This model predicts the conductive temperature structure above a specific isotherm $T_{\text {lit }}$ which represents the bottom of the lithosphere; here we choose $T_{\text {lit }}=$ $1603 \mathrm{~K}$. Initial temperatures below this isotherm are calculated by linear interpolation between $T_{\text {lit }}$ and $T_{\text {bot }}$. We also used a plate model with a thermal thickness of $90 \mathrm{~km}$ to test the sensitivity of the results to the initial conditions. As expected, the half-space model results in a faster and more efficient thermal erosion of the plate in the first $60 \mathrm{Ma}$ of simulation compared to the plate model, which is intrinsically more stable. However, since our calculations are continued until a statistical steady-state is achieved, results are not affected by the assumed initial temperature field. We apply free slip boundary conditions along the sides and bottom of the box and no slip at the top, where a constant horizontal velocity of $3.5 \mathrm{~cm} \mathrm{a}^{-1}$ is imposed.
[6] We calculate the (static) boundary layer contribution to seafloor topography as

$$
w_{\text {iso }}=\frac{1}{\left(\rho_{\text {ref }}-\rho_{w}\right)} \int_{0}^{d_{\text {com }}}\left(\rho-\rho_{\text {ref }}\right) \mathrm{d} z
$$

where $d_{\text {com }}$ is a compensation depth and $\rho_{\text {ref }}$ is the depth dependent density of a reference column. Although the choice of $d_{\text {com }}$ is somewhat arbitrary, it should not be taken deeper than the deepest isotherm defining the base of the thermal boundary layer ( $\sim 1600 \mathrm{~K}$ isotherm). Deeper dynamic loads associated with SSC are not expected to be locally compensated and their dynamic contribution to topography is only minor $(< \pm 150 \mathrm{~m})$.

[7] Following Afonso et al. [2007], the extent of melting $F$ experienced by ascending mantle at the MOR is approximated with two linear functions intercepting at an appropriate depth, marking the point where the melt productivity function $\gamma=(d F / d P)_{S}$ changes its slope. Different pressures at which partial melting begins $\left(P_{0}\right)$ are analyzed. The final $\mathrm{H}_{2} \mathrm{O}$ content of the solid residue is estimated directly from the melting model through the $\mathrm{H}_{2} \mathrm{O}$ partition coefficient (see auxiliary material). We assume that when a mantle parcel reaches $F=\xi \%$ partial melting ( $\xi$ varies between 2 and $5 \%$ in different experiments; see below), it can be effectively considered as "dry" mantle. The associated viscosity increase due to dehydration is computed as $\eta_{\text {eff }}=\eta_{\text {wet }} W_{\mathrm{H}_{2}} \mathrm{O}+$ $\eta_{\text {dry }}\left(1-W_{H_{2}} O\right)$, where $W_{H_{2}} O$ takes the values 1,0 , or $(1-$ $F / \xi$ ) when $F$ is $0, \xi$, or $0<F<\xi$, respectively. $\eta_{\text {wet }}$ is calculated using the "wet" parameters listed in Table S1 (auxiliary material) considering both diffusion and dislocation creep mechanisms. $\eta_{d r y}$ is assumed to be either $50 \eta_{\text {wet }}$ or $100 \eta_{\text {wet }}$ [Karato and Wu, 1993; Hirth and Kohlstedt, 1996], depending on the experiment.

[8] The olivine $\alpha-\beta$ and $\beta-\gamma$ transitions at $\sim 410$ and $520 \mathrm{~km}$ are incorporated in our models. For the former, we assign a 10-fold viscosity increase. The spinel-garnet solid phase transition is modeled as a function of $T$ and $P$ according to the experimental results of Klemme and $O$ 'Neill [2000], with the corresponding density jump taken from Afonso et al. [2007]. Since there are still significant uncertainties associated with the depth of spinel-garnet phase change, we also run models varying this depth. Inasmuch as the plagioclase-spinel phase transition occurs at depths that are too shallow $(\sim 30 \mathrm{~km})$ to be affected by or have an effect on SSC, it is not included in our calculations.

\section{Results}

[9] In keeping with our goal of generating models consistent with experimental/observational data, we chose not to use parameterized rheological laws (e.g., Frank-Kamenetskii) or "customized" creep parameters, as commonly done in the literature. Instead, we use experimentally derived sets of creep parameters in combination with the same flow laws employed to obtain them from deformation experiments (see auxiliary material). We tested two popular sets of creep parameters: one is from Karato and $W u$ [1993] and the other is from Hirth and Kohlstedt [2003]. For each set we run identical simulations in which we vary the melting model $\left(P_{0}\right.$ and $\left.\xi\right)$ and the depth of the spinel-garnet transition. We 

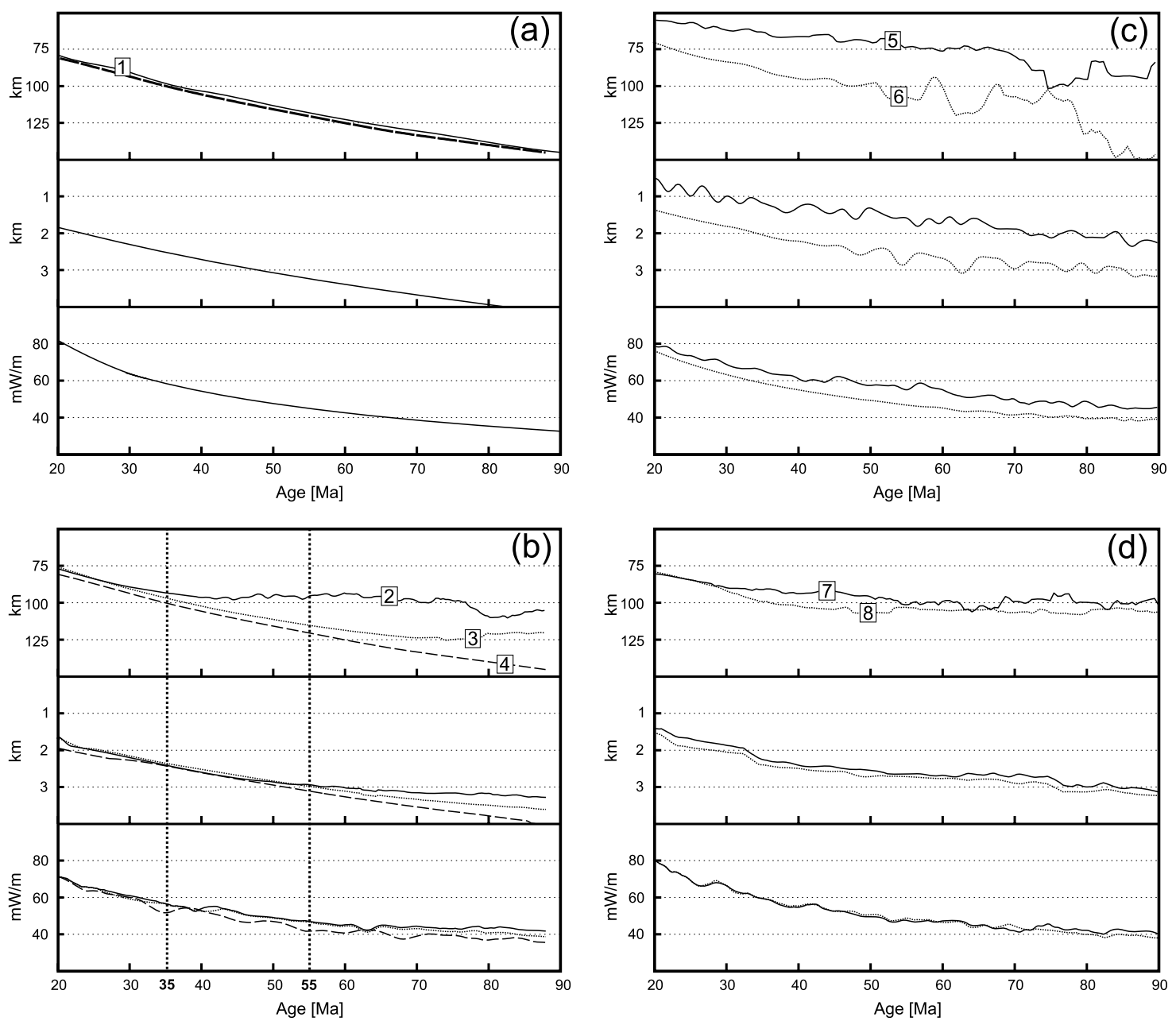

Figure 2. (top) Age-dependent lithospheric thickness, (middle) bathymetry, and (bottom) SHF for 4 different sets of experiments. The short-wavelength $(\$ 200 \mathrm{~km})$ component of SSC is removed from the isotherm defining the base of the thermal lithosphere by applying a short-wavelength smoothing filter. Bathymetry is calculated relative to the depth of the ridge crest. (a) Simulation 1, representative results form simulations with "dry" rheology. The dashed line represents predictions from the half-space cooling model. (b) Simulations in which melting begins at depths of $80 \mathrm{~km}(2)$, $110 \mathrm{~km}$ (3), and $150 \mathrm{~km}$ (4). Dotted vertical lines denote the SSC onset times of simulations 2 and 3. (c) Identical simulations with (6) and without (5) adiabatic heating. (d) Simulations with the spinel-garnet phase change taking place at $80 \mathrm{~km}(7)$ and $40 \mathrm{~km}(8)$ depth.

find that when using the values reported by Karato and $W u$ [1993] and Hirth and Kohlstedt [2003] for "dry" rheology (Table S1), simulations predict average upper mantle viscosities $\gtrsim 10^{20} \mathrm{~Pa} \mathrm{~s}$ (Figure $1 \mathrm{~b}$ ). These values are found to be high enough to inhibit the development of SSC, and consequently both the thermal structure of the OL and geophysical observables follow closely those predicted by a half-space cooling model (Figure 2a). Although some small instabilities may develop in the older parts of the OL, they cannot evolve into well defined drippings due to the high viscosity of the underlying mantle. These observations concur with the detailed study of Zlotnik et al. [2008] on the rheological control of SSC. If the values reported by Hirth and Kohlstedt [2003] for a constant water content of $1000 \mathrm{H} /$ $10^{6} \mathrm{Si}$ are used instead (more in agreement with the actual water content of the MORB source), the average viscosity of the upper mantle drops to values of $10^{19}-10^{20} \mathrm{~Pa}$ (Figure 1a), and SSC becomes active. The final viscosity structure obtained with these parameters is almost identical to that obtained with the set of Karato and Wu [1993] for "wet" creep (Table S1), which also generate SSC.

[10] The inclusion of melting in our "wet" simulations generates a strong correlation between the assumed melting model and the development and vigour of SSC, which in turn makes the final thermal structure of the OL dependent on the history of melting at the MOR. In models in which melting starts at depths between $\sim 60$ and $140 \mathrm{~km}$, we find that higher $\mathrm{P}_{0}$ values (i.e., melting begins deeper) result in thicker plates due to the stabilizing effect of the viscosity increase related to dehydration. Shallower melting models generates the high-viscosity zone related to dehydration well within the already highly viscous part of the OL resulting from the normal conductive cooling. In these cases, melting do not play any role in either the development of SSC or in the final thermal structure of the plate. On the other hand, deeper melting models tend to sluggish or 


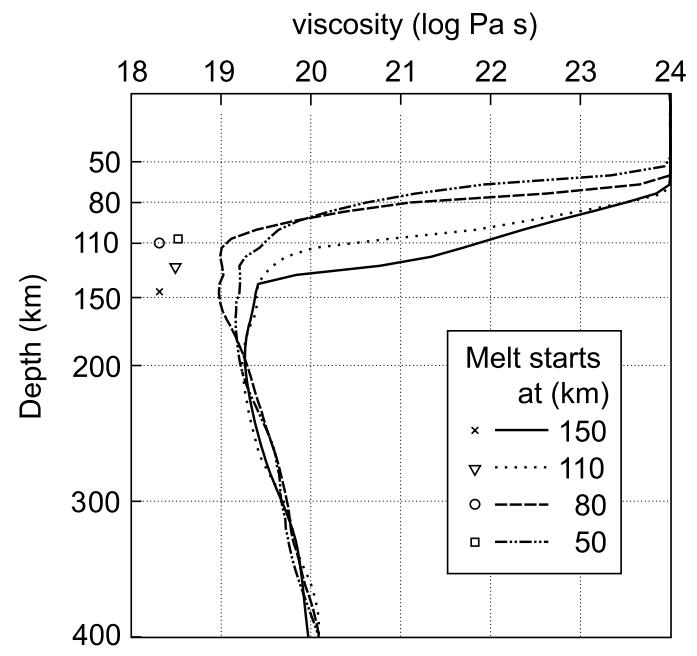

Figure 3. Effective viscosity profiles from simulations in which we vary the depth (strictly, $P_{0}$ ) at which partial melting begins (see also Figure $2 b)$. Symbols $(\times, \nabla, \bigcirc, \square)$ indicate the steady-state thermal thickness (i.e., depth to the $1603 \mathrm{~K}$ isotherm) resulting from these simulations when the plate is $\sim 90$ Ma old.

even suppress SSC due to the higher developed viscosities (Figure 3). The dependence of SSC onset times on melting models, as speculated by Lee et al. [2005], is evident in Figure $2 \mathrm{~b}$. Note also that in models where a flattening of the signals occur (e.g., simulation 2 in Figure 2b), a clear time delay exists between the onsets of SSC and the flattening. This time delay is a measure of the time needed by SSC to flatten out the conductive cooling of the plate. Varying $\xi$ from 2 to $5 \%$ and $\eta_{d r y}$ from $50 \eta_{\text {wet }}$ to $100 \eta_{\text {wet }}$ has a very minor effect on the above results.

[11] Previous studies have demonstrated the important role of radiogenic heat production in controlling the thermal thickness of OL and the cooling of the sublithospheric upper mantle by SSC [e.g., Jarvis and Peltier, 1980; Huang and Zhong, 2005; Zlotnik et al., 2008], and therefore we do not address this point here. We note, however, that all our simulations include a realistic radiogenic heat production value of $1.8 \times 10^{-8} \mathrm{~W} \mathrm{~m}^{-3}$ [Labrosse and Jaupart, 2007]. The role of the adiabatic heating term, on the other hand, is not always well appreciated. Neglecting this term increases the density difference between upwellings and downwellings, and consequently the vigour and eroding capacity of SSC (Figure 2c). This may result in underestimations of the steady-state thermal thickness of OL by as much as $25-$ $50 \mathrm{~km}$ [see also Zlotnik et al., 2008].

[12] Figure 2d illustrates that the final thermal structure of the OL is relatively insensitive to the inclusion (and variation) of the spinel-garnet phase transition. Parallel simulations (not shown here) demonstrate that the same applies for the inclusion of compositional density stratification generated by partial melting at the MOR. This occurs because density differences between the depleted residue and the undepleted mantle become significant only at depths $\$ 30 \mathrm{~km}$, where partial melting $>8-10 \%$ [Afonso et al., 2007]. These depths are too shallow to influence SSC. Regarding the role of the phase transition, we note that although new laboratory experiments could modify somewhat its thermodynamic behaviour, its effect on SSC is not expected to change significantly. Although both compositional stratification and phase changes are unimportant for $\mathrm{SSC}$, they do control the total buoyancy of the plate [Afonso et al., 2007].

\section{Discussion and Conclusions}

[13] The results confirm that SSC is a feasible process in the oceanic mantle and compatible with experimental creep data on "wet" olivine aggregates. They also demonstrate that under most circumstances, the viscosity increase due to mantle dehydration at MORs has first-order effects in the vigour of SSC, on its ability to thermally erode the OL, and hence on determining its final thermal structure and associated geophysical observables (Figure 2b). Unfortunately, one must acknowledge that dynamic systems such as the one described in this work are extremely dependent on their viscosity structure [e.g., Zlotnik et al., 2008], which in turn depends on experimental parameters that are subject to large uncertainties. This partially may be the cause why other studies on SSC under oceans failed to slow down seafloor subsidence and produce a near constant surface heat flow [Korenaga and Jordan, 2002]. We find that manipulating creep parameters within uncertainties to favour large temperature and strain-rate dependences in dislocation creep may also result in a flattening of seafloor and surface heat flow without invoking dehydration effects. In this case, mantle viscosity at $\sim 90-100 \mathrm{~km}$ depth evolves spontaneously to reach peak values $\lesssim 10^{18} \mathrm{~Pa} s$ in the dislocation regime, in which case SSC results in an OL with a nearly constant thermal thickness (and signal flattening) defined by the depth at which the viscosity minimum is reached $(\sim 90-$ $100 \mathrm{~km}$ ). However, the resulting average viscosity seems to be too low to be compatible with estimations from sea-mount loading [Watts and Zhong, 2000]. Also, if melting begins deeper than this depth, the dehydration effect would still control the final structure of the plate (Figures $2 b$ and 3 ).

[14] We find that models that successfully reproduce geophysical observables are also characterized by a significant stress-dependent (i.e., dislocation) creep component on their effective viscosities in the first 200-250 km (for grain sizes $d \geq 4 \mathrm{~mm}$; Figure $1 \mathrm{a}$ and auxiliary material), with peaks between 120 and $150 \mathrm{~km}$. The participation of dislocation creep at these depths not only enhances the vigour of SSC and the flattening of the signals, but also implies the existence of seismic anisotropy in this region. This is consistent with recent seismic anisotropy studies in the oceanic upper mantle that find significant horizontal anisotropy between 50 and $250 \mathrm{~km}$ depth, with a clear peak around $125 \mathrm{~km}$ depth [Nettles and Dziewonski, 2008].

[15] Acknowledgments. We thank M. Ballmer, G. Ranalli and W. Griffin for valuable comments on an earlier version of this paper. We are also grateful for the constructive reviews by S. Karato and L. Moresi. This work was supported by the projects CTM2005-08071-C03-03/MAR and Consolider TopoIberia CSD2006-00041.

\section{References}

Afonso, J. C., G. Ranalli, and M. Fernàndez (2007), Density structure and buoyancy of the oceanic lithosphere revisited, Geophys. Res. Lett., 34, L10302, doi:10.1029/2007GL029515. 


\section{AFONSO ET AL.: SMALL-SCALE CONVECTION BENEATH OCEAN BASINS}

Ballmer, M. D., J. van Hunen, G. Ito, P. J. Tackley, and T. A. Bianco (2007), Non-hotsop volcano chains originating from small-scale sublithospheric convection, Geophys. Res. Lett., 34, L23310, doi:10.1029/ 2007 GL031636.

Davaille, A., and C. Jaupart (1994), Onset of thermal convection in fluids with temperature-dependent viscosity: Application to the oceanic mantle, J. Geophys. Res., 99, 19,853-19,866.

Dumoulin, C., M.-P. Doin, and L. Fleitout (2001), Numerical simulations of the cooling of an oceanic lithosphere above a convective mantle, Phys. Earth Planet. Inter., 125, 45-64.

Dumoulin, C., M. Doin, D. Arcay, and L. Fleitout (2005), Onset of smallscale instabilities at the base of the lithosphere: Scaling laws and role of pre-existing lithospheric structures, Geophys. J. Int., 160, 344-356.

Hillier, J. K., and A. B. Watts (2005), Relationship between depth and age in the North Pacific Ocean, J. Geophys. Res., 110, B02405, doi:10.1029/ 2004JB003406.

Hirth, G., and D. L. Kohlstedt (1996), Water in the oceanic upper mantle: Implications for rheology, melt extraction and the evolution of the lithosphere, Earth Planet. Sci. Lett., 144, 93-108.

Hirth, G. and D. L. Kohlstedt (2003), Rheology of the upper mantle and the mantle wedge: A view from the experimentalists, in Inside the Subduction Factory, Geophys. Monogr., vol. 138, edited by J. Eiler, pp. 83-105, AGU, Washington, D. C.

Huang, J., and S. Zhong (2005), Sublithospheric small-scale convection and its implications for the residual topography at old ocean basins and the plate model, J. Geophys. Res., 110, B05404, doi:10.1029/2004JB003153.

Huang, J., S. Zhong, and J. van Hunen (2003), Controls on sublithospheric small-scale convection, J. Geophys. Res., 108(B8), 2405, doi:10.1029/ 2003JB002456.

Jarvis, G., and W. Peltier (1980), Oceanic bathymetry profiles flattened by radiogenic heating in a convecting mantle, Nature, 285, 649-651.

Karato, S. I., and P. Wu (1993), Rheology of the upper mantle: A synthesis, Science, 260, 771-778.
Klemme, S., and H. S. O’Neill (2000), The near-solidus transition from garnet lherzolite to spinel lherzolite, Contrib. Mineral. Petrol., 138, 237248.

Korenaga, J., and T. H. Jordan (2002), On "steady-state" heat flow and rheology of oceanic mantle, Geophys. Res. Lett., 29(22), 2056, doi:10.1029/2002GL016085

Labrosse, S., and C. Jaupart (2007), Thermal evolution of the Earth: Secular changes and fluctuations of plate characteristics, Earth Planet. Sci. Lett., 260, 465-481.

Lee, C.-T. A., A. Lenardic, C. M. Cooper, F. Niu, and A. Levander (2005), The role of chemical boundary layers in regulating the thickness of continental and oceanic thermal boundary layers, Earth Planet. Sci. Lett., 230, 379-395.

Nettles, M., and A. Dziewonski (2008), Radially anisotropic shear-velocity structure of the upper mantle globally and beneath norht america, J. Geophys. Res., 113, B02303, doi:10.1029/2006JB004819.

Phipps Morgan, J. (1997), The generation of a compositional lithosphere by mid-ocean ridge melting and its effect on subsequent off-axis hotspot upwelling and melting, Earth Planet. Sci. Lett., 146, 213-232.

Solomatov, S., and L.-N. Moresi (2000), Scaling of time dependent stagnant lid convection: Application to small-scale convection on Earth and other terrestrial planets, J. Geophys. Res., 105, 12,795-21,817.

Watts, A., and S. Zhong (2000), Observations of flexure and the rheology of oceanic lithosphere, Geophys. J. Int., 142, 855-875.

Zlotnik, S., J. C. Afonso, P. Díez, and M. Fernàndez (2008), Small-scale gravitational instabilities under the oceans: Implications for the evolution of the oceanic lithosphere and its expression in geophysical observables, Philos. Mag. A, in press.

J. C. Afonso, M. Fernàndez, and S. Zlotnik, GDL, Institute of Earth Sciences “J. Almera," CSIC, Luís Solé i Sabarís s/n, ES-08028 Barcelona, Spain. (jafonso@ija.csic.es; mfernandez@ija.csic.es; szlotnik@ija.csic.es) 